\title{
Gene expression profiling and molecular pathway analysis for the identification of early-stage lung adenocarcinoma patients at risk for early recurrence
}

\author{
HISASHI SAJI $^{1}$, MASAHIRO TSUBOI ${ }^{1}$, YOSHIHISA SHIMADA ${ }^{1}$, YASUFUMI KATO ${ }^{1}$, \\ WAKAKO HAMANAKA ${ }^{1}$, YUJIN KUDO ${ }^{1}$, KOICHI YOSHIDA ${ }^{1}$, JUN MATSUBAYASHI ${ }^{2}$, \\ JITSUO USUDA ${ }^{1}$, TATSUO OHIRA ${ }^{1}$ and NORIHIKO IKEDA ${ }^{1}$ \\ ${ }^{1}$ Division of Thoracic Surgery, Department of Surgery, and ${ }^{2}$ Department of Anatomic Pathology, \\ Tokyo Medical University, Shinjuku-ku, Tokyo 160-0023, Japan
}

Received November 2, 2012; Accepted January 16, 2013

DOI: 10.3892/or.2013.2332

\begin{abstract}
Clinicohistopathological staging is insufficient to predict disease progression and clinical outcome in lung carcinoma. Based on the results of the principal component analysis of 24 samples of early-stage lung adenocarcinoma, two subgroups were identified within the early-relapse group. The histological classification of all samples of group A was poorly differentiated, whereas one out of three in group B was poorly differentiated. DAVID functional annotation analysis revealed that the molecular pathways enriched in group A included those associated with cell adhesion molecules (CAMs), cell cycle and antigen processing and presentation, whereas those in group B included CAMs, T cell receptor signaling, cytokine-cytokine receptor interaction, toll-like receptor signaling, chemokine signaling, primary immunodeficiency and natural killer cell-mediated cytotoxicity. The CAM pathway was enriched in both groups. This comprehensive gene expression and functional pathway analysis identified a distinct molecular pathway, CAMs, that correlated with the early relapse of patients with early-stage lung adenocarcinoma.
\end{abstract}

\section{Introduction}

Lung cancer is the leading cause of cancer-related deaths in Japan and also worldwide in most developed countries. Every year, 60,000 individuals succumb to lung cancer in Japan, and the number is increasing rapidly. Even in early-stage lung cancer, $\sim 40 \%$ of patients with stage I and II non-small cell lung cancer (NSCLC) die from recurrent disease within 5 years despite complete resection $(1,2)$. The precise diagnosis

Correspondence to: Dr Hisashi Saji, Division of Thoracic Surgery, Department of Surgery, Tokyo Medical University, 6-7-1 Nishishinjuku, Shinjuku-ku, Tokyo 160-0023, Japan

E-mail: saji-q@ya2.so-net.ne.jp

Key words: lung adenocarcinoma, prognostic factor, microarray, molecular pathway analysis, early relapse, cell adhesion molecule and classification of cancers are critical for the selection of appropriate therapies. However, since no reliable clinical or molecular predictors are currently available, it is difficult to select high-risk patients who require more aggressive therapies such as adjuvant chemotherapy.

Genetic abnormalities that exist in a certain population of early-stage lung cancer patients possibly induce aggressive phenotypes that demonstrate rapid tumor growth, persistent invasiveness and a high potential for distant metastasis. The expression of a number of genes is altered in cancer cells due to mutations, deletions, amplifications, and either the upregulation or downregulation of mRNA transcription. Comprehensive DNA microarray analysis of gene expression patterns is a powerful tool that permits the simultaneous evaluation of a large number of genes in cancer cells $(3,4)$. Microarray gene expression profiling has recently been used to define prognostic signatures in patients with NSCLC (5-11). However, information concerning gene expression profiling and molecular pathways relating to the outcomes of patients with early-stage lung cancer has yet to be well characterized.

Adenocarcinoma is currently the predominant histological subtype of NSCLC. The results of several expression profiling studies have demonstrated that the expression profiles are distinctive and recapitulate the known histological subtypes (5-7). As a significant proportion of patients relapse within 2 years, identification of early-stage patients with a poor prognosis could delineate the appropriate candidates for adjuvant therapy. The present study aimed to identify a novel prognostic signature in early-stage lung adenocarcinoma using cDNA microarray and bioinformatics analysis.

\section{Materials and methods}

Patient samples. Intraoperatively, immediately upon removal of a lung lobe in which a primary lung carcinoma was located, a 500-mg sample of tumor tissue was cut and immediately immersed in liquid nitrogen and stored at $-80^{\circ} \mathrm{C}$ until use, as previously reported (12). We studied frozen specimens of lung cancer tissue from 64 randomly selected patients who underwent complete resection of stage I or II NSCLC lesions 
at Tokyo Medical University, Tokyo, Japan from May 2003 to December 2006. Tumor tissues were processed by the Human Tissue Bank section at our department according to standard operating procedures and protocols. Briefly, frozen tissue samples at $-80^{\circ} \mathrm{C}$ were pulverized, and total cellular RNA was collected from each flash-frozen sample using TRIzol RNA isolation reagent (Invitrogen). Total RNA was processed with an RNeasy Mini kit (Qiagen). In vitro transcription-based RNA amplification was then performed on at least $8 \mu \mathrm{g}$ of total RNA from each sample. The RNA quality was assessed using a bioanalyzer (model 2100, Agilent). According to the results from the RNA quality assay, 24 lung adenocarcinoma samples were selected as our dataset.

Microarray analysis. Complementary DNA was synthesized using the T7-(dT)24 primer: 59-GGCCAGTGAATTGTA ATACGACTCACTATAGGGAGGCGG-(dT)24-39. The cDNA was processed using phase-lock gel phenol/chloroform extraction (\#E0032005101, Fisher). Next, in vitro transcriptional labeling with biotin was performed using the Enzo BioArray kit (\#900182, Affymetrix). The resulting cRNA was processed again using the RNeasy Mini kit. Labeled cRNA was hybridized to an Affymetrix GeneChip (Human Genome-133 Plus 2.0 Array) according to the manufacturer's instructions. The raw fluorescence intensity data within the CEL files were preprocessed with the robust multichip average algorithm, as implemented with the $\mathrm{R}$ packages from Bioconductor. This algorithm analyzes the microarray data in three steps: a background adjustment, quantile normalization, and finally summation of the probe intensities for each probe set using a log scale linear additive model for the log transform of (background corrected, normalized) PM intensities.

Data analysis. Affymetrix Human Genome-U133 Plus 2.0 GeneChip data, quantified with MAS5, were imported into the Subio Platform (Subio Inc., Tokyo, Japan). Signals $<1$ were replaced with $1, \log 2$ transformed, and then mean-subtracted by each probe set to obtain the log ratio against the average of the expression patterns. No normalization was applied.

Samples were classified into two groups, recurrencepositive and recurrence-negative. Probe sets in both groups whose detection values were absent in half of the samples were removed. At this point, 28674 out of 54682 probe sets remained. Finally, unvarying probe sets, whose log ratios were between -1 and +1 in all samples, were filtered out to obtain the final quality controlled probe sets (24420).

Principal component analysis (PCA) was applied to the log ratio data of quality controlled genes. We recognized that the samples in the recurrence-positive group might be distinguishable as PC1 score negative (A) and positive (B) subgroups.

We extracted the differentially expressed genes (DEGs) for both A and B subgroups. We defined DEGs for A as being $>4$-fold upregulated or downregulated compared with the average of the recurrence-negative group, and having Mann-Whitney U-test P-values of $<0.05$ between the recurrence-negative group and the recurrence-positive A subgroup. A total of 721 probe sets were selected as DEG for A. Similarly, we obtained 274 probe sets as DEGs for B, which showed a $>2$-fold change and P-values of $<0.05$ by the Mann-Whitney U-test, as compared with the recurrence-negative group.
Biological analysis of the DEG lists. We searched 171 and 33 enriched GO terms for DEGs determined for the A and B group, respectively, with the annotation analysis plug-in of the Subio platform (data not shown). We further analyzed these lists with the DAVID functional annotation web tool (http:// david.abcc.ncifcrf.gov) and obtained the lists of enriched KEGG pathways (Tables I and II).

Ethical considerations. Written informed consent was obtained from the patients for tissue procurement prior to surgery and their medical records were maintained according to protocols approved by the Institutional Review Board of Tokyo Medical University (no. 965).

\section{Results}

Patient information. As shown in Table I, there were 14 male and 10 female patients enrolled in this study. The mean age was 65.3 years (range, 42-76). The histological classifications were all adenocarcinoma; 14 were well/moderately differentiated and 10 were poorly differentiated. The distribution of clinical staging demonstrated that most of the patients were early-stage IAB cases. Histological differentiation was significantly correlated with early recurrence $(\mathrm{P}=0.026)$, whereas no significant correlations were found among pathological stages IA, IB and IIA $(\mathrm{P}=0.061)$.

Correlation of patient outcome with putative adenocarcinoma classes. We aimed to ascertain whether lung cancer patient outcome correlates with the subclasses of lung adenocarcinomas defined herein. Based on the results of PCA of this series, two adenocarcinoma subgroups were identified within the early-relapse group of early-stage adenocarcinoma cases, which differentially expressed a broad range of gene patterns (Fig. 1).

Statistical analysis of the microarray data, when compared with the non-early-relapse group $\mathrm{C}$, revealed 723 genes with significant differences in expression in the samples of group A, whereas 274 genes showed significant differences in expression in samples of group B. We searched 171 and 33 altered GO terms for DEGs in the A and B lists, respectively, with the annotation analysis plug-in of the Subio platform (data not shown).

The histological classification of all samples of group A was poorly differentiated, whereas only one out of three cases in group B was classified as poorly differentiated. In this series of early-stage IA-IIA adenocarcinomas, no papillary or bronchio-alveolar carcinoma subtypes were associated with recurrence within 2 years after complete resection.

Biological function analysis. Tables II and III document the 16 and 17 enriched pathways in groups $\mathrm{A}$ and $\mathrm{B}$, respectively. Clusters of genes related to oncological or immunological functional signaling were found enriched in group A as were pathways such as cell adhesion molecules (CAMs), cell cycle, and antigen processing and presentation. In group B, the pathways included CAMs, T cell receptor signaling, cytokinecytokine receptor interaction, toll-like receptor signaling, chemokine signaling pathway, primary immunodeficiency and natural killer cell mediated cytotoxicity. The CAM pathway was found to be enriched in both groups A and B. 
Table I. Patient information regarding the 24 adenocarcinoma samples.

\begin{tabular}{|c|c|c|c|c|}
\hline \multirow[b]{2}{*}{ Variables } & \multirow[b]{2}{*}{ Total samples } & \multicolumn{2}{|c|}{ Early recurrence } & \multirow[b]{2}{*}{ P-value } \\
\hline & & Positive $(\mathrm{n}=8)$ & Negative $(n=16)$ & \\
\hline Median age (range) & $65.3(42-76)$ & $67.0(55-76)$ & $64.5(42-76)$ & 0.551 \\
\hline Gender & & & & 0.143 \\
\hline Male & 14 & 3 & 11 & \\
\hline Female & 10 & 5 & 5 & \\
\hline Histological differentiation & & & & $0.026^{\mathrm{a}}$ \\
\hline Well/moderate & 14 & 2 & 11 & \\
\hline Poor & 10 & 6 & 4 & \\
\hline p-Stage & & & & 0.061 \\
\hline IA & 14 & 2 & 12 & \\
\hline IB & 8 & 5 & 3 & \\
\hline IIA & 2 & 1 & 1 & \\
\hline
\end{tabular}

aSignificant difference.

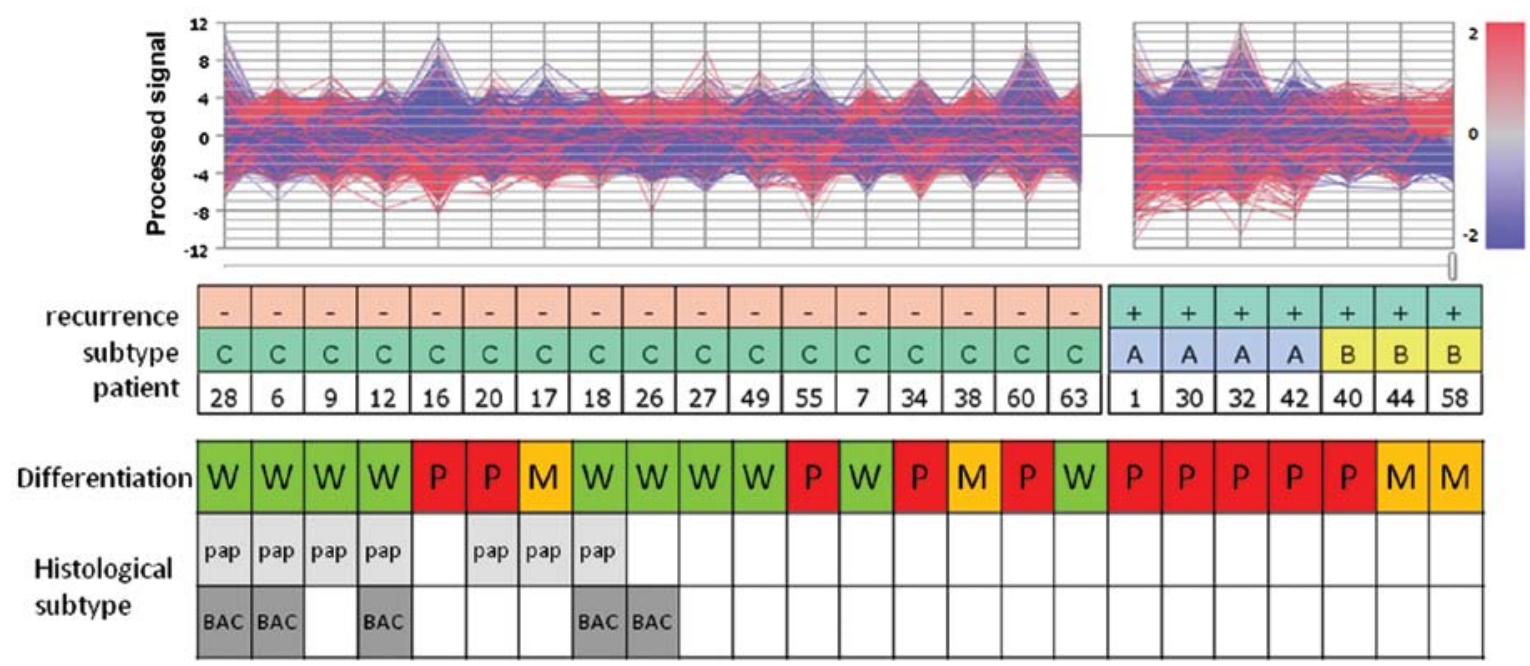

Figure 1. Principal component analysis correlated with differentiation and histological subtype. W, well differentiated; M, moderately differentiated; P, poorly differentiated; BAC, bronchio-alveolar carcinoma; pap, papillary carcinoma.

\section{Discussion}

The development of microarray technologies has made it possible to quantitate the expression of many thousands of genes simultaneously in a given sample $(3,4)$. Comprehensive analysis of gene expression patterns in individual tumors should, therefore, provide detailed molecular portraits that can facilitate tumor classification. Several expression profiling studies concluded that expression profiles are distinctive and recapitulate known histological subtypes (5-7).

Genomic methods offer promise for the classification of human lung carcinomas. In one previous study, it is important to note that the performance of the adenocarcinoma classifier showed a better predictive accuracy than the squamous cell lung carcinoma (SCC) classifier (adenocarcinoma $\mathrm{AUC}=0.83$, SCC AUC $=0.68$ ). This could have been due to the hetero- geneity of the SCC samples as indicated by the two distinct subgroups showing differing clinical outcomes in this tumor type (9). Multiple independent studies of mRNA expression profiles in lung adenocarcinoma have proven highly reproducible. Analyses of the relationship between expression profiles and tumor development and differentiation, the presence or absence of specific pathogenic mutations, patient prognosis and survival after surgical treatment, and specific histopathology all appear to be promising (13).

Adenocarcinoma is currently the predominant histological subtype of NSCLC. NSCLC composes the majority of bronchogenic carcinoma cases with a lesser fraction being small-cell lung carcinomas. The three main subtypes of NSCLC are adenocarcinoma (60\%), SCC (25\%) and large-cell cancer (5\%). Adenocarcinoma has replaced SCC as the most frequent histological subtype over the last 25 years $(1,2,14)$. Therefore, 
Table II. Enriched pathways in group A.

\begin{tabular}{llrrrc}
\hline Category & \multicolumn{1}{c}{ Term } & Count & $\%$ & P-value & Benjamini \\
\hline KEGG_PATHWAY & Asthma & 8 & 1.5 & 0.000019 & 0.0024 \\
KEGG_PATHWAY & Allograft rejection & 8 & 1.5 & 0.000085 & 0.0053 \\
KEGG_PATHWAY & Graft-versus-host disease & 8 & 1.5 & 0.00014 & 0.0061 \\
KEGG_PATHWAY & Cell adhesion molecules (CAMs) & 14 & 2.5 & 0.00017 & 0.0052 \\
KEGG_PATHWAY & Type I diabetes mellitus & 8 & 1.5 & 0.00023 & 0.0059 \\
KEGG_PATHWAY & Intestinal immune network for IgA production & 8 & 1.5 & 0.00062 & 0.013 \\
KEGG_PATHWAY & Autoimmune thyroid disease & 8 & 1.5 & 0.0008 & 0.014 \\
KEGG_PATHWAY & Hematopoietic cell lineage & 10 & 1.8 & 0.0011 & 0.017 \\
KEGG_PATHWAY & Systemic lupus erythematosus & 10 & 1.8 & 0.003 & 0.041 \\
KEGG_PATHWAY & Antigen processing and presentation & 8 & 1.5 & 0.013 & 0.15 \\
KEGG_PATHWAY & PPAR signaling pathway & 7 & 1.3 & 0.018 & 0.19 \\
KEGG_PATHWAY & Oocyte meiosis & 9 & 1.6 & 0.018 & 0.18 \\
KEGG_PATHWAY & Viral myocarditis & 7 & 1.3 & 0.02 & 0.18 \\
KEGG_PATHWAY & Arachidonic acid metabolism & 6 & 1.1 & 0.027 & 0.22 \\
KEGG_PATHWAY & Cell cycle & 9 & 1.6 & 0.036 & 0.27 \\
KEGG_PATHWAY & Drug metabolism & 6 & 1.1 & 0.04 & 0.27 \\
\hline
\end{tabular}

Table III. Enriched pathways in group B.

\begin{tabular}{llrrrc}
\hline Category & \multicolumn{1}{c}{ Term } & Count & $\%$ & P-value & Benjamini \\
\hline KEGG_PATHWAY & T cell receptor signaling pathway & 11 & 4.8 & 0.0000046 & 0.00048 \\
KEGG_PATHWAY & Cytokine-cytokine receptor interaction & 16 & 7 & 0.0000075 & 0.00039 \\
KEGG_PATHWAY & Toll-like receptor signaling pathway & 9 & 3.9 & 0.00014 & 0.0047 \\
KEGG_PATHWAY & Cell adhesion molecules (CAMs) & 10 & 4.3 & 0.00016 & 0.0042 \\
KEGG_PATHWAY & Leukocyte transendothelial migration & 8 & 3.5 & 0.0021 & 0.041 \\
KEGG_PATHWAY & Chemokine signaling pathway & 10 & 4.3 & 0.0021 & 0.035 \\
KEGG_PATHWAY & Intestinal immune network for IgA production & 5 & 2.2 & 0.0064 & 0.091 \\
KEGG_PATHWAY & Autoimmune thyroid disease & 5 & 2.2 & 0.0074 & 0.091 \\
KEGG_PATHWAY & Primary immunodeficiency & 4 & 1.7 & 0.016 & 0.17 \\
KEGG_PATHWAY & Type I diabetes mellitus & 4 & 1.7 & 0.026 & 0.24 \\
KEGG_PATHWAY & Axon guidance & 6 & 2.6 & 0.047 & 0.36 \\
KEGG_PATHWAY & Cytosolic DNA-sensing pathway & 4 & 1.7 & 0.052 & 0.37 \\
KEGG_PATHWAY & Natural killer cell mediated cytotoxicity & 6 & 2.6 & 0.053 & 0.35 \\
KEGG_PATHWAY & NOD-like receptor signaling pathway & 4 & 1.7 & 0.069 & 0.41 \\
KEGG_PATHWAY & Focal adhesion & 7 & 3 & 0.087 & 0.46 \\
KEGG_PATHWAY & RIG-I-like receptor signaling pathway & 4 & 1.7 & 0.095 & 0.47 \\
KEGG_PATHWAY & Prion diseases & 3 & 1.3 & 0.1 & 0.47 \\
\hline
\end{tabular}

we focused on adenocarcinoma of the lung, and particularly whether we could identify a novel prognostic signature of early recurrence in early-stage lung adenocarcinoma using cDNA microarray techniques.

The data indicated that patterns of gene expression obtained from cDNA microarray studies of crudely dissected lung tumors can be used to detect tumor subtypes that correlate with biological and clinical phenotypes. Specifically, patterns of gene expression were found that corresponded to the major morphological classes of lung tumors. In addition, we were able to define two subgroups of early recurrence in the adenocarcinoma cases that differed not only in gene expression patterns, but also in clinical and pathological properties, including histological differentiation and subtype. In the statistical analysis of microarray data, when compared with the non-early-recurrence group $\mathrm{C}$, we revealed 723 genes with significant differences in expression in the samples of group A, whereas 274 genes showed significant differences in 
expression in group B. The differentially expressed genes were classified according to biological processes. We searched 171 and 33 enriched GO terms for DEGs for the A and B lists, respectively, with the annotation analysis plug-in of the Subio platform (data not shown).

Gene annotation enrichment analysis is a functional analysis technique that has gained widespread attention and for which many tools have been developed. The differentially expressed genes were classified according to biological processes and molecular functions using the functional annotation clustering tool of the DAVID bioinformatics resources. The DAVID functional clustering analysis revealed 16 significantly altered biological pathways in group A that included 3 distinct functionally related metastatic categories, specifically CAMs, cell cycle, and antigen processing and presentation. In group B, there were 17 significantly altered biological pathways, including 7 distinct functionally related metastatic categories. Notably, the CAM pathway was the most interrelated in both groups. In addition, the $\mathrm{T}$ cell receptor signaling pathway, cytokine-cytokine receptor interaction, toll-like receptor signaling pathway, chemokine signaling pathway, primary immunodeficiency and natural killer cell mediated cytotoxicity were also altered (Tables II and III). These results suggest that the possibility of metastasis of earlystage lung adenocarcinoma was closely related to the CAM pathway. Interestingly, considering the relationship between group A or group B and histological differentiation as poor or well/moderate, respectively, the metastatic possibility of poorly differentiated early adenocarcinoma appeared to be correlated with tumor development factors, such as the cell cycle, whereas that of well/moderately differentiated earlystage adenocarcinoma appeared to be correlated with host immunological factors, such as the $\mathrm{T}$ cell receptor signaling pathway, cytokine-cytokine receptor interaction, the toll-like receptor signaling pathway, the chemokine signaling pathway, primary immunodeficiency and natural killer cell mediated cytotoxicity.

Our results suggest that the particular genes that define the clusters and molecular pathways, or that are associated with early recurrence, likely reflect the characteristics of the particular tumors included in the analysis. Current therapy for patients with early-stage disease usually consists of surgical resection without adjuvant treatment. Clearly, the identification of a high-risk group among early-stage patients would lead to consideration of additional therapeutic interventions, possibly leading to improved survival of these patients.

To our knowledge, this is the first study utilizing cDNA microarray techniques, followed by molecular functional pathway analysis, concerning the early recurrence of earlystage adenocarcinoma of the lung. However, there were some limitations to this study. Firstly, this was a small data set analysis at a single institute. A large cohort sample of patients from multiple institutions is needed. Secondly, the potential interactions of the many specific individual genes and their clusters in lung tumor biology and clinical outcome exist. This may be due to the different platforms used (different genes analyzed) and the different algorithms for selecting functional categories. Thirdly, hierarchical clustering methods and functional analysis offer a powerful approach to class discovery, but provide no means of determining validity for the classes discovered. This is still a putative functional analysis. It is important to state that several in vitro and in vivo studies are still needed to demonstrate whether these mechanisms are effective in reality.

In conclusion, in the present study, we present a comprehensive gene expression analysis and functional pathway analysis of early-stage lung adenocarcinomas, wherein we identified a distinct molecular pathway category, the CAMs, which correlated with the early relapse of early-stage lung adenocarcinoma subclasses. Further in vitro and in vivo studies, which can demonstrate these mechanisms, are warranted.

\section{Acknowledgements}

We are indebted to Dr Clifford A. Kolba, to Associate Professor Edward F. Barroga and to Professor J. Patrick Barron, Chairman of the Department of International Medical Communications of Tokyo Medical University, for their editorial review of the English manuscript. This study was supported by grants from the Ministry of Education, Culture, Sports, Science and Technology (grant no. 21791332).

\section{References}

1. Sawabata N, Asamura H, Goya T, et al: Japanese Lung Cancer Registry Study: first prospective enrollment of a large number of surgical and nonsurgical cases in 2002. J Thorac Oncol 5: 1369-1375, 2010.

2. Asamura H, Goya T, Koshiishi Y, et al: A Japanese Lung Cancer Registry study: prognosis of 13,010 resected lung cancers. J Thorac Oncol 3: 46-52, 2008

3. Schena M, Shalon D, Davis RW and Brown PO: Quantitative monitoring of gene expression patterns with a complementary DNA microarray. Science 270: 467-470, 1995.

4. Chee M, Yang R, Hubbell E, et al: Accessing genetic information with high-density DNA arrays. Science 274: 610-614, 1996.

5. Beer DG, Kardia SL, Huang CC, et al: Gene-expression profiles predict survival of patients with lung adenocarcinoma. Nat Med 8: 816-824, 2002.

6. Bhattacharjee A, Richards WG, Staunton J, et al: Classification of human lung carcinomas by mRNA expression profiling reveals distinct adenocarcinoma subclasses. Proc Natl Acad Sci USA 98: 13790-13795, 2001.

7. Garber ME, Troyanskaya OG, Schluens K, et al: Diversity of gene expression in adenocarcinoma of the lung. Proc Natl Acad Sci USA 98: 13784-13789, 2001.

8. Wigle DA, Jurisica I, Radulovich N, et al: Molecular profiling of non-small cell lung cancer and correlation with disease-free survival. Cancer Res 62: 3005-3008, 2002.

9. Raponi M, Zhang Y, Yu J, et al: Gene expression signatures for predicting prognosis of squamous cell and adenocarcinomas of the lung. Cancer Res 66: 7466-7472, 2006.

10. Lu Y, Yao R, Yan Y, et al: A gene expression signature that can predict green tea exposure and chemopreventive efficacy of lung cancer in mice. Cancer Res 66: 1956-1963, 2006.

11. Potti A, Mukherjee S, Petersen R, et al: A genomic strategy to refine prognosis in early-stage non-small-cell lung cancer. $\mathrm{N}$ Engl J Med 355: 570-580, 2006.

12. Nakamura H, Saji H, Ogata A, et al: cDNA microarray analysis of gene expression in pathologic stage IA nonsmall cell lung carcinomas. Cancer 97: 2798-2805, 2003.

13. Meyerson M and Carbone D: Genomic and proteomic profiling of lung cancers: lung cancer classification in the age of targeted therapy. J Clin Oncol 23: 3219-3226, 2005.

14. Sawabata N, Miyaoka E, Asamura $\mathrm{H}$, et al: Japanese lung cancer registry study of 11,663 surgical cases in 2004: demographic and prognosis changes over decade. J Thorac Oncol 6: 1229-1235, 2011. 